\title{
Flocculation in Schizosaccharomyces pombe
}

\author{
By G. B. CALLEJA \\ Biochemistry Laboratory, National Research Council of Canada, Ottawa, Canada
}

(Accepted for publication 28 October 1970)

Spontaneous formation of macroscopic aggregates of yeast cells is termed flocculation. Many papers have been written on the subject (for reviews see Comrie, 1952, Gilliland, 1957; Jansen, 1958; Rainbow, 1966; Windisch, 1968), but because of the importance of the phenomenon to the brewer most of the studies have been confined to brewer's yeast. An analogous phenomenon in a fission yeast is described here.

Schizosaccharomyces pombe NCYC I32 was grown in I $25 \mathrm{ml}$. Erlenmeyer flasks each containing $10 \mathrm{ml}$. malt-extract broth (Oxoid, $2 \%$, w/v). The inoculum consisted of $5 \times 10^{6}$ stationary-phase yeast cells from a $24 \mathrm{~h}$. static culture in the same medium in a tightly capped McCartney bottle. Cultures were shaken on a rotatory shaker at $\mathrm{I} 50 \mathrm{rev} . / \mathrm{min}$. at $32^{\circ}$; the generation time was $120 \mathrm{~min}$.

\section{RESULTS AND DISCUSSION}

Flocculation, operationally defined as the formation of aggregates consisting of more than Io organisms, occurred only during the stationary growth phase. Observed with a microscope, the first flocs appeared 2 to $3 \mathrm{~h}$. after the end of logarithmic growth. Maximum flocculation was attained within 5 to Io h., when flocs contained 25 to $35 \%$ of the population. The largest floc contained up to $10^{5}$ organisms. Static cultures, such as those used for inocula, did not flocculate even when incubated for weeks, provided they remained largely anaerobic. Organisms grown in a medium containing (per 1.) 5 g. yeast extract (Difco) +30 g. glucose, static or shaken, never flocculated.

Formation of flocs was not due to failure of daughter organisms to separate after fission. An hour or two before flocculation, aggregates consisting of more than Io organisms were never observed. Moreover, separation of pairs and larger aggregates by ultrasonic treatment at the end of logarithmic growth did not prevent subsequent flocculation.

Flocs were stable to dilution in deionized water and could be separated from free (non-flocculated) organisms by differential sedimentation. A flocculated culture was transferred to a $15 \mathrm{ml}$. tapered centrifuge tube and allowed to stand undisturbed for $5 \mathrm{~min}$. at room temperature. The flocs settled to the bottom of the tube, whereas free organisms remained in the supernatant fluid which could then be decanted from the flocs or sampled in situ for counting with a haemocytometer. Flocs were purified by repeated sedimentation in deionized water. Free organisms separated from flocs during the time of maximum flocculation could not be induced to flocculate among themselves when shaken in their own medium and thus were considered to be noninduced or incompetent.

That flocs were stable in deionized water indicates that no special component of spent medium sustained floc formation. Addition of uninoculated medium, or of 
spent medium separated from logarithmically growing cultures, to purified flocs did not cause deflocculation. This observation indicates that the failure of growing organisms to flocculate was not due to the presence of a deflocculating agent in the medium.

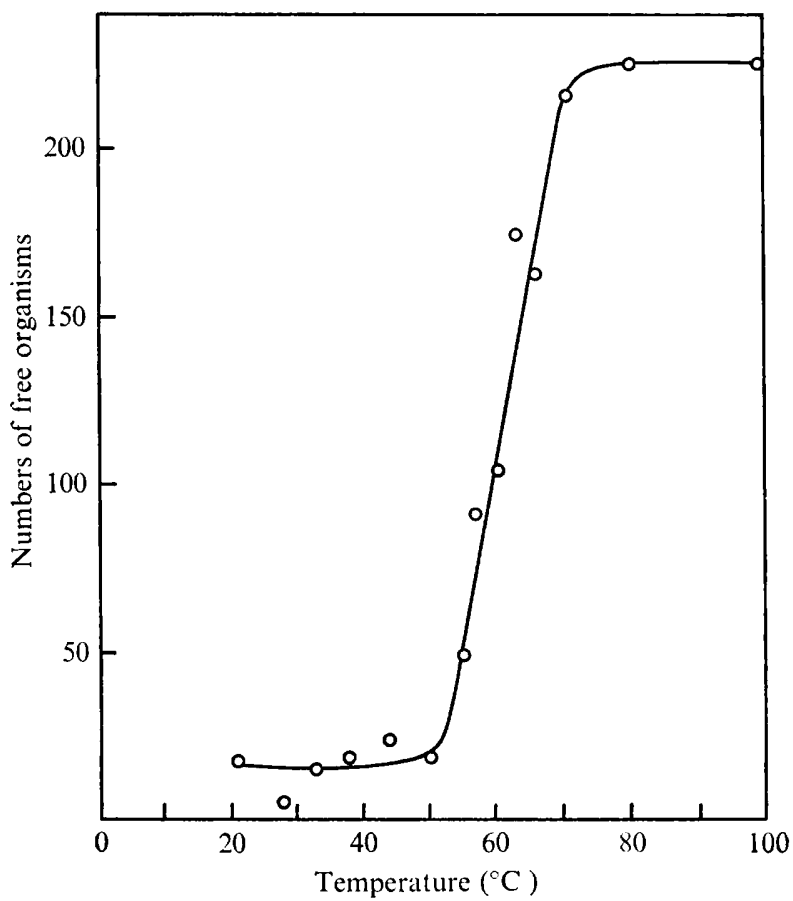

Fig. I. Effect of temperature on the stability of purified flocs of Schizosaccharomyces pombe NCYC 132. The extent of deflocculation is expressed as the number of free organisms in the suspensions observed microscopically.

The effect of temperature on the stability of flocs in deionized water is shown in Fig. I. A ro ml. suspension of purified flocs in a tapered centrifuge tube was incubated in a water bath, and the temperature of the bath was raised $I^{\circ}$ every $5 \mathrm{~min}$. Counts of free organisms were made as follows. The tube was withdrawn, inverted five times, and returned to the bath to remain undisturbed until sampled. At the end of 5 min., the supernatant fluid was sampled for counting, $10 \mathrm{~mm}$. below the liquid surface. Only organisms in groups of Io or less were counted. Purified flocs were stable to $50^{\circ}$; higher temperatures caused deflocculation, which was complete at $80^{\circ}$ and above. The temperature at which half the floc population deflocculated was $6 \mathrm{I}^{\circ}$. A roughly similar value has been reported for brewer's yeast (Mill, 1964).

Deflocculation by heat was reversible. When a tube of heat-deflocculated organisms was allowed to stand undisturbed at room temperature, organisms slowly reflocculated. The time taken for half the population to reflocculate $\left(R_{\mathrm{t}}\right)$ was ro to I $5 \mathrm{~h}$. Continuous gentle agitation shortened $R_{\mathrm{t}}$ values to an hour or so. Addition of $0.1 \mathrm{M}$ salt, such as $\mathrm{NaCl}$ or $\mathrm{CaCl}_{2}$, decreased the $R_{\mathrm{t}}$ value to a few minutes.

Calcium ions have been directly implicated in the flocculation of brewer's yeast (Mill, I964) and in other cell-aggregating systems (for a review, see Curtis, 1967). I 
found no evidence that $\mathrm{Ca}^{2+}$ were directly involved in flocculation of this fission yeast Schizosaccharomyces pombe. The stability of purified flocs in deionized water and the ineffectiveness of EDTA (pH 8) as a deflocculating agent are evidence against the involvement of $\mathrm{Ca}^{2+}$. Moreover, repeated washing of deflocculated organisms with deionized water did not inhibit subsequent reflocculation. Furthermore, the use of salts to increase the rate of reflocculation could be obviated by packing the organisms by centrifugation. Then, upon resuspension, the organisms were seen to have reflocculated. Incompetent organisms sedimented in a centrifuge did not flocculate.

Because the population of free organisms could be determined from a sample of the supernatant fluid, and the total population estimated after deflocculation, it was possible to calculate the number of flocculated organisms in a culture without isolating the flocs. A culture whose population of free organisms had been sampled was deflocculated at $90^{\circ}$. After cooling to room temperature, it was sampled for a total count. The number of organisms in flocs was then equivalent to the total population determined after deflocculation minus the free organism population determined after standing for $5 \mathrm{~min}$. and before deflocculation.

Incubation of purified flocs in $3 \mathrm{M}$-guanidinium chloride or in $5 \mathrm{M}$-urea caused complete deflocculation, an observation that has been made with brewer's yeast (Mill, 1964). Removal of either reagent led to instantaneous reflocculation. Sodium dodecyl sulphate also caused reversible, but very slow, deflocculation. In contrast, deflocculation by trypsin or papain was irreversible, in the same sense that the effect of neither reagent could be removed by washing. The use of proteolytic enzymes as disaggregating agents for brewer's yeast (Eddy, 1958) and other cell-aggregating systems (for a review, see Curtis, I967) is well known. Sugars, reported to cause deflocculation of brewer's yeast (Eddy, I955; Mill, I964) were ineffective even at concentrations as high as $50 \%$ $(w / v)$. High concentrations of $\mathrm{NaCl}$ were likewise ineffective.

Since heat-killed organisms reflocculated, incompetent organisms and organisms in flocs were separately ruptured by means of a Braun cell homogenizer. The envelopes were separated from cellular debris and whole organisms by differential centrifugation and washed repeatedly with deionized water. Envelopes derived from organisms in flocs flocculated; those from incompetent organisms did not. Thus whatever forces were involved in floc formation must have been associated with the envelopes.

Reflocculation of organisms reversibly deflocculated by any of the ways described above showed significant hysteresis. The highest reflocculation value observed was $95 \%$.

The author thanks Dr Byron F. Johnson and Dr Ian J. McDonald for helpful criticism and advice.

NRC contribution no. II 778.

\section{REFERENCES}

CoMrie, A. D. D. (1952). Yeast flocculation. Wallerstein Laboratories Communications 15, 339-345. Curtis, A.S. G. (1967). The Cell Surface: its Molecular Role in Morphogenesis. London: Academic Press.

EDDY, A. A. (1955). Flocculation characteristics of yeasts. II. Sugars as dispersing agents. Journal of the Institute of Brewing 6r, 3I3-3I 7.

EDDY, A. A. (1958). Part of the yeast surface apparently involved in flocculation. Journal of the Institute of Brewing 64, 19-21. 
GILlILAND, R. B. (1957). The flocculation of brewing yeast. Wallerstein Laboratories Communications 20, 4I-7I.

JANSEN, H. E. (1958). Flocculation of yeasts. In The Chemistry and Biology of Yeasts, pp. 635-667. Edited by A. H. Cook. London: Academic Press.

MILL, P. J. (1964). The nature of the interaction between flocculent cells in the flocculation of Saccharomyces cerevisiae. Journal of General Microbiology 35, 6I-68.

RaINBow, C. (1966). Flocculation of brewer's yeast. Process Biochemistry I, 489-492.

WINDISCH, S. (I968). Flocculation of brewing yeasts. Brewer's Digest 43, 62-66. 